\title{
Design of a Ka-band microwave undulator
}

\author{
Liang Zhang ${ }^{1,2}$, Wenlong He ${ }^{1}$, Jim Clarke ${ }^{2,3}$, Kevin Ronald ${ }^{1,2}$, Alan D.R. Phelps ${ }^{1}$ and Adrian W. Cross ${ }^{1,2}$ \\ ${ }^{1}$ Department of Physics, SUPA, University of Strathclyde, Glasgow, G4 0NG, Scotland, UK \\ ${ }^{2}$ Cockcroft Institute, Warrington Bank Quay, WA4 4AD UK. \\ ${ }^{3}$ ASTeC, STFC Daresbury Laboratory, Sci-Tech Daresbury, Warrington, WA4 4AD, UK.
}

\begin{abstract}
In this paper, a microwave undulator operating at $36 \mathrm{GHz}$ was proposed and design for a UK XFEL. A corrugated waveguide which has low loss was used. The properties of the microwave undulator operating at $\mathrm{HE}_{11}$ and $\mathrm{HE}_{12}$ modes are compared.
\end{abstract}

Keywords-microwave undulator, free electron laser, corrugated waveguide, balanced hybrid condition.

\section{INTRODUCTION}

Undulators that to provide a periodic transverse magnetic field are important components in a free electron laser (FEL) [1, 2]. Compared with the permanent magnet undulator, the microwave undulators [3, 4] have the advantages of its larger beam aperture and adjustable field strength and the polarization. [5]. The microwave undulator is essentially a cavity structure. By operating higher frequency, it is able to achieve smaller period. However, for permanent magnet undulator, it is challenging because it will significantly reduce the field strength. The microwave undulator is also robust against damage by the ionizing radiation as compared with the permanent magnet undulators that are made of rare earth materials.

The successful operation of a microwave undulator requires the cavity to have a high quality factor. In a circular waveguide, the $\mathrm{TE}_{01}$ mode has the least loss. However, the biggest field strength of $\mathrm{TE}_{01}$ mode is off centered. Therefore it is not an ideal option for the microwave undulator. The $\mathrm{HE}_{11}$ mode in a corrugated wave has been proved to be a low loss mode as well. It has been widely used as the feed horn and the transmission line for a high power gyrotron. A microwave undulator that composed by a cavity made of a corrugated waveguide has been experimentally studied. It operated at X-band and achieved a high $Q$ factor up to 91000 . An equivalent magnetic field of 0.65 $\mathrm{T}$ was achieved when it was driven by a 50 MW SLAC klystron [6]. The period of the microwave undulator was $13.9 \mathrm{~mm}$.

The FEL can achieve radiation with smaller wavelength if the wavelength of the undulator can be smaller. In this paper, a microwave undulator operating at a higher frequency of $36 \mathrm{GHz}$ was proposed and designed for a UK XFEL.

\section{DESIGN OF THE CORRUGATED WAVEGUIDE}

The schematic drawing of a corrugated waveguide is shown in Fig. 1. It contains circular waveguide steps and smooth sections. The properties of a corrugated waveguide with arbitrary radial corrugation depth can be accurately solved using a mode-matching method [7-9]. For the periodically corrugated waveguide, theoretical studied based on the balanced hybrid condition has also been derived to get an analytical solution of the dispersion curve [10-13].

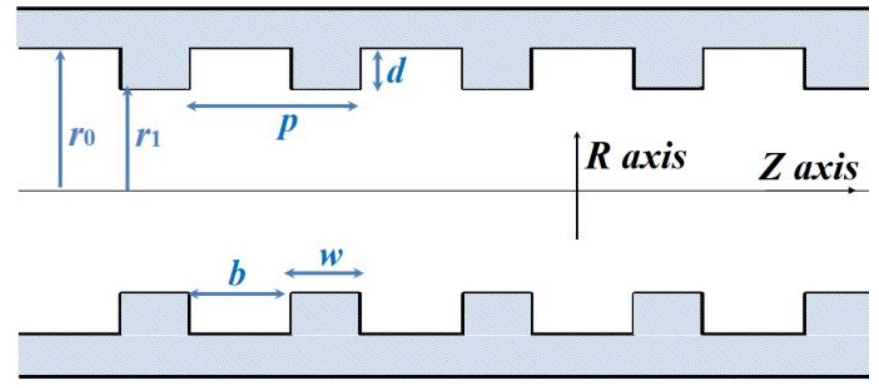

Fig. 1. Schematic drawing of the corrugated waveguide.

The most suitable operating modes in the corrugated waveguide as a microwave undulator are the $\mathrm{HE}_{11}$ and $\mathrm{HE}_{12}$ modes. They are low loss and have maximum field strength at the waveguide center. They are also linearly polarized therefore the beam dynamic inside the waveguide is easier to understand. Fig. 2 shows the electric field patterns of the $\mathrm{HE}_{11}$ and $\mathrm{HE}_{12}$. At the same operating frequency, the $\mathrm{HE}_{12}$ can have much larger waveguide radius.

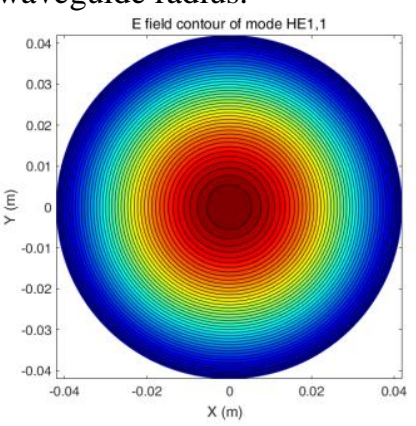

(a)

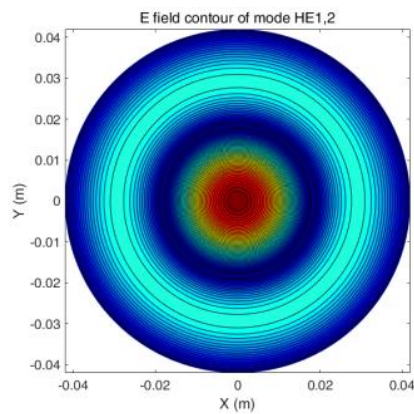

(b)
Fig. 2. Contour plot of the electric field patterns of the $\mathrm{HE}_{11}$ and $\mathrm{HE}_{12}$ modes.

The initial geometry of the corrugated waveguide can be derived from the field pattern and the dispersion relation. As a comparison, Table 1 listed the parameters of the microwave undulator for the $\mathrm{HE}_{11}$ and $\mathrm{HE}_{12}$ modes, operating at $36 \mathrm{GHz}$.

TABLE 1 Parameters of the MU composed of a corrugated waveguide.

\begin{tabular}{|l|l|l|}
\hline Operating mode & $\mathrm{HE}_{11}$ & $\mathrm{HE}_{12}$ \\
\hline Operating frequency $(\mathrm{GHz})$ & 36 & 36 \\
\hline$\lambda_{0}(\mathrm{~mm})$ & 8.33 & 8.33 \\
\hline
\end{tabular}




\begin{tabular}{|l|l|l|}
\hline$R_{b}(\mathrm{~mm})$ & 2.0 & 2.0 \\
\hline$r_{1}(\mathrm{~mm})$ & $4 R_{b}=8.0$ & $9 R_{b}=18.0$ \\
\hline$d=\lambda_{0} / 4(\mathrm{~mm})$ & 2.1 & 2.1 \\
\hline$\lambda_{g}(\mathrm{~mm})$ & 9.06 & 9.12 \\
\hline$p=\lambda_{g} / 3 \quad(\mathrm{~mm})$ & 3.00 & 3.02 \\
\hline$w(\mathrm{~mm})$ & 0.5 & 0.5 \\
\hline$b=p-w(\mathrm{~mm})$ & 2.50 & 2.52 \\
\hline
\end{tabular}

\section{REFERENCES}

[1] D. A. G. Deacon, L. R. Elias, J. M. J. Madey, G. J. Ramian, H. A Schwettman, and T. I. Smith, "First Operation of a Free-Electron Laser," Phys. Rev. Lett., vol. 38, no. 16, pp. 892-894, April 1977. DOI: 10.1103/PhysRevLett.38.892

[2] Z. Huang and K.-J. Kim, "Review of x-ray free-electron laser theory," Physical Review Special Topics - Accelerators and Beams, vol. 10, no. 3, p. 034801, March 2007. DOI: 10.1103/PhysRevSTAB.10.034801

[3] S. Tsumoru, H. Kazuo, T. Jiro, S. Isamu, and K. Isao, "Development of Microwave Undulator," Jpn. J. Appl. Phys., vol. 22, no. 5R, pp. 844-851, May 1983. DOI: 10.1143/JJAP.22.844

The eigen frequency and quality factor of the designed structure were further simulated using the eigen solver in the CST microwave studio. The undulator used in a FEL is usually a long structure. Here a 6-period structure was simulated to reduce the simulation time. The dimensions were slightly adjusted to match the resonance frequency of $36 \mathrm{GHz}$.

Instead of the electric conductive boundary condition, a periodic boundary condition was set at both ends of the corrugated waveguide. In this way, the loss at both sides will not be included therefore it will not affect much on the quality factor value. It was found that with the same input power and similar operating frequency, the waveguide radius of the $\mathrm{HE}_{12}$ mode was about 2.2 times that of the $\mathrm{HE}_{11}$ mode. And its $\mathrm{Q}$ factor was nearly two times that of the $\mathrm{HE}_{11}$ mode. However, the equivalent magnetic field was similar for both cases. The $\mathrm{HE}_{12}$ mode has the advantage of larger dimensions at a higher frequency and easier to be machined. However, as operating at higher order mode, the input coupler will be more complicated.

A longer structure of the microwave undulator, which contains 100 periods as well as the coupler at both sides, operating at $\mathrm{HE}_{11}$ mode was also simulated. The electric field pattern is shown in Fig. 3. It has a very uniform electric field along the axial direction and the quality factor is larger than 70000 in the simulation.

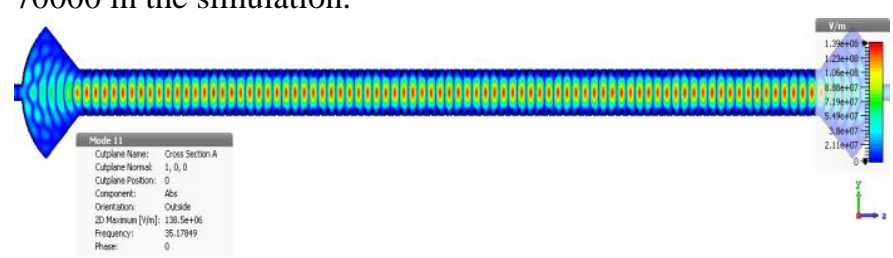
Undulator," Japanese Journal of Applied Physics, vol. 21, no. 10A, p. L601, 1982. http://stacks.iop.org/1347-4065/21/i=10A/a=L601

M. Shumail and S. G. Tantawi, "Theory of electromagnetic insertion devices and the corresponding synchrotron radiation," Physical Review Accelerators and Beams, vol. 19, no. 7, p. 074001, 07/27/ 2016.

https://link.aps.org/doi/10.1103/PhysRevAccelBeams.19.074001

[6] S. Tantawi, M. Shumail, J. Neilson, G. Bowden, C. Chang, E Hemsing, and M. Dunning, "Experimental Demonstration of a Tunable Microwave Undulator," Phys. Rev. Lett., vol. 112, no. 16, p. 164802, April 2014. DOI: 10.1103/PhysRevLett.112.164802

[7] G. L. James, "Analysis and Design of $\mathrm{TE}<\mathrm{sub}>11</$ sub $>$-to$\mathrm{HE}<\mathrm{sub}>11</ \mathrm{sub}>$ Corrugated Cylindrical Waveguide Mode Converters," IEEE Trans. Microwave Theory Techn., vol. 29, no. 10, pp. 1059-1066, 1981. DOI: 10.1109/TMTT.1981.1130499

[8] J. M. Neilson, P. E. Latham, M. Caplan, and W. G. Lawson, "Determination of the resonant frequencies in a complex cavity using the scattering matrix formulation," IEEE Trans. Microwave Theory Techn., vol. 37, no. 8, pp. 1165-1170, 1989. DOI: $10.1109 / 22.31074$

[9] L. Zhang, W. He, C. R. Donaldson, and A. W. Cross, "Bandwidth Study of the Microwave Reflectors with Rectangular Corrugations," Journal of Infrared, Millimeter, and Terahertz Waves, journal article vol. 37, no. 9, pp. 846-856, September 01 2016. DOI 10.1007/s10762-016-0280-y

[10] P. J. B. Clarricoats and A. D. Olver, Corrugated Horns for Microwave Antennas. Institution of Engineering and Technology, 1984.

[11] C. Dragone, "Attenuation and Radiation Characteristics of the HE /Sub 11/ - Mode," IEEE Trans. Microwave Theory Techn., vol. 28, no. 7, pp. 704-710, 1980. DOI: 10.1109/TMTT.1980.1130152 P. J. B. Clarricoats and P. K. Saha, "Propagation and radiation behaviour of corrugated feeds. Part 2: Corrugated-conical-horn feed," Electrical Engineers, Proceedings of the Institution of, vol. 118, no. 9, pp. 1177-1186, 1971. DOI: 10.1049/piee.1971.0212

[13] E. J. Kowalski, D. S. Tax, M. A. Shapiro, J. R. Sirigiri, R. J. Temkin, T. S. Bigelow, and D. A. Rasmussen, "Linearly Polarized Modes of a Corrugated Metallic Waveguide," IEEE Trans. Microwave Theory Techn., vol. 58, no. 11, pp. 2772-2780, 2010. DOI: 10.1109/TMTT.2010.2078972

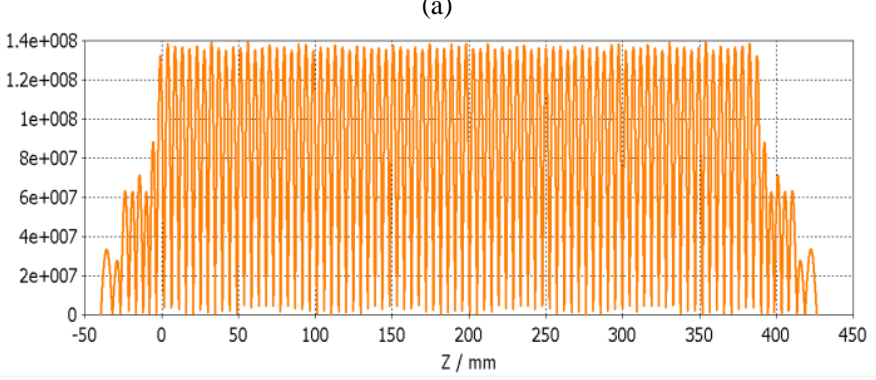

(b)

Fig. 3. Field pattern inside of the corrugated waveguide (a), and the Ex component of the field (b).

\section{ACKNOWLEDGMENT}

This work is under the support of Science and Technology Facilities Council (STFC) U.K. 